\title{
Chiral physics in the magnetic field with quark confinement contribution
}

\author{
M.A. Andreichikov ${ }^{\mathrm{a}} \mathbb{0}$, Yu. A. Simonov \\ State Research Center, Institute of Theoretical and Experimental Physics, Moscow 117218, Russia
}

Received: 9 August 2018 / Accepted: 27 October 2018 / Published online: 8 November 2018

(C) The Author(s) 2018

\begin{abstract}
The standard chiral perturbation theory is known to predict much weaker effects in magnetic field, than found in numerical lattice data. To overcome this disagreement we are using the effective chiral confinement Lagrangian, $L_{E C C L}$, containing both chiral and quark degrees of freedom, in the presence of external magnetic field. Without magnetic fields $L_{E C C L}$ reduces to the ordinary chiral Lagrangian $L_{E C L}$, yielding in the lowest order $O\left(\partial_{\mu} \varphi\right)^{2}$ all known relations, and providing explicit numerical coefficients in the higher $O\left(p^{4}, p^{6}\right)$ orders. The inclusion of the magnetic field in $L_{E C C L}$ strongly modifies ECL results for chiral condensates, coupling constants $f_{\pi}, f_{K}$ and masses of chiral mesons. The resulting behavior contains the only parameter - the string tension $\sigma$, is roughly proportional to $O\left(\frac{e B}{\sigma}\right)$ and agrees very well with lattice data. These results show that the magnetic field acts not only on the chiral degrees of free$\operatorname{dom}\left(\varphi_{\pi}\right)$, but also on quarks in the quark-chiral Lagrangian, which produce much stronger effect.
\end{abstract}

\section{Introduction}

The effective chiral theory (ECL) [1-3] was developing long before the notion of the quark dynamics was introduced, and the relation between the quark confinement and dynamics and ECL remains obscure to a large extent till now.

In particular, the leading terms of the effective chiral Lagrangian (ECL) [4-6] to all orders do not contain any relation to quark dynamics and quark masses appear actually as correction terms.

Nevertheless even in this form ECL was very useful to describe low energy reactions with chiral mesons. At higher energies, where internal structure of chiral mesons becomes more important, one needs additional power terms $O\left(p^{4}, p^{6}\right)$ and some modifications of the theory, and the corresponding

a e-mail: andreichicov@mail.ru effective models with adjustable coefficients have been suggested, [7-9], which can describe the experimental data.

So far so good, but at some moment an interesting idea has appeared, what could happen, when one considers the chiral systems in the constant magnetic field (m.f.). As an immediate reaction it was suggested, that one can use as an effective Lagrangian in m.f. the well known ECL with the replacement of the derivatives $\left(\partial_{\mu} U\right)^{n}$ in its terms by the full derivative $D_{\mu} U=\partial_{\mu} U+i e A_{\mu} U$ which takes into account the action of m.f. on the chiral d.o.f. in $U=\exp \left(\frac{i \hat{i}_{a} \lambda_{a}}{f_{\pi}}\right)$.

This idea was considered well substantiated and it was used in many papers, both in the ECL [10-16] and in the NJL model [18-20].

In particular, for the quark condensate in [10] with the definition $\langle\bar{q} q\rangle \equiv \sum=|\langle\bar{u} u\rangle|=|\langle\bar{d} d\rangle|$ it was found, using chiral perturbation theory (ChPT)

$$
\sum(B)=\sum(0)\left[1+\frac{e B \ln 2}{16 \pi^{2} F_{\pi}^{2}}+O\left(\frac{e B}{16 \pi^{2} f_{\pi}^{2}}\right)^{2}\right] .
$$

Note the integer value of charge $e$ in (1), and the same linear behavior for $\langle\bar{u} u\rangle$ and $\langle\bar{d} d\rangle$ condensates, which is the consequence of purely chiral degrees of freedom, having only integer charges, but looks unrealistic for the quark observable with charge $e_{q} \neq e$. In [10] also the relation for $f_{\pi^{0}}$ and $M_{\pi_{0}}$ were found, supported later with corrections due to $m_{\pi}^{2} \neq 0$ in [11-17]

$$
\begin{aligned}
& \frac{f_{\pi^{0}}^{2}(B)}{f_{\pi^{0}}^{2}(0)}=1+\frac{e B \ln 2}{8 \pi^{2} f_{\pi}^{2}}+\cdots \\
& M_{\pi^{0}}^{2}(B)=M_{\pi^{0}}^{2}(0)\left[1-\frac{e B \ln 2}{16 \pi^{2} f_{\pi}^{2}}+\cdots\right]
\end{aligned}
$$

The appearance of the factor $1 / f_{\pi}^{2}$ in (1), (2), (3) is not surprising, since this is the basic dimensional factor in the ECL.

$L=\frac{f_{\pi}^{2}}{4} \operatorname{tr}\left(\partial_{\mu} U \partial_{\mu} U^{+}\right)+O\left(\hat{m}_{q} U\right)+\cdots$ 
The idea of a specific chiral-magnetic physics, mostly independent of separate quark degrees of freedom, was and still is attractive and produced several directions. One of this is the theory suggested in [21].

One assumes in this type of approach that quark d.o.f. are inessential for low values of $e B \ll\left(4 \pi f_{\pi}\right)^{2}$, where the phenomena of the chiral magnetic system can be observed, while for higher m.f. as was argued in [10] the $q \bar{q}$ d.o.f. can be important, but due to asymptotic freedom quarks can be considered as free of strong interaction and only subject to m.f. Thus one can neglect strong interactions, and first of all the confinement, considering chiral systems, made of quarks, in weak and strong m.f. However, the accurate lattice calculations of the quark condensate $|\langle\bar{q} q\rangle|$ in [22-25] have provided much stronger linear growth of $|\langle\bar{q} q\rangle|$, than in the ChPT [10]. In general, the numerous lattice data in [22-32] demonstrate much stronger influence of m.f. on chiral observables, than is predicted by $\mathrm{ChPT}$.

It is a purpose of this paper to present a theory and explicit calculations which can explain this disagreement. We take into account quarks and antiquarks with confinement in the framework of the Effective Chiral Confinement Lagrangian (ECCL) [33-41] in m.f. and demonstrate, that confinement yields much larger effects due to m.f., than in the purely chiral theory shown in (1), (2), (3).

We also show that our results are in good agreement with available lattice data [22-32] for the same physical quantities.

To understand the importance of confinement in the magnetic effects of chiral observables one can compare the pure chiral results (1), (2), (3), which can be written as $X_{\Sigma, f, M}=$ $1+a_{\Sigma, f, M}^{(C h P T)} \frac{e B}{\left(4 \pi f_{\pi}\right)^{2}}+\cdots$, where $a_{\Sigma, f, M}=O(1)$, which means that chiral observables are essentially controlled by the parameter $\left(4 \pi f_{\pi}\right)^{2} \sim O\left(1 \mathrm{GeV}^{2}\right)$.

This is to be compared with the effects of m.f. with the confinement interaction between $q$ and $\bar{q}$, which has the order of magnitude $O\left(\frac{e B}{\sigma}\right)$, with the standard string tension $\sigma=$ $0.18 \mathrm{GeV}^{2}$. In particular, for neutral pion decay constant $f_{\pi^{0}}$ one obtains, $\left(\frac{f_{\pi^{0}}(e B)}{f_{\pi^{0}}(0)}\right)=\sqrt{1+\left(\frac{e_{q} B}{\sigma}\right)^{2}}$.

Moreover, the confinement is the basic interaction which allows to calculate all coefficients in the ECL including the orders $O\left(p^{4}\right)$ and $O\left(p^{6}\right)$ as it is shown in [37].

Therefore one expects that the theory of chiral observables based on both quark and chiral d.o.f. should provide a more appropriate set up for the calculation of all quantities $\Sigma, f, M$ for both neutral and charged pions in m.f. are in good agreement with lattice data [22-32]. As will be seen, our formalism provides numerical results for all observables $\Sigma, f, M$ without fitting parameters in the few percent agreement with all lattice data.

The paper is organised as follows. In the next section we start from the ECCL Lagrangian and derive all three chiral observables $\Sigma, f, M$ without m.f. taking into account both chiral and quark d.o.f. We pay below a special attention to the separation of purely $q \bar{q}$ and chiral d.o.f. and discuss also higher order corrections. In Sect. 3 we discuss the effects of m.f. on chiral and confinement dynamics and obtain $\Sigma, f, M$ in the presence of m.f. In Sect. 4 we shortly demonstrate the results of ECCL calculations for $\Sigma, f, M$ in m.f. and compare those with lattice data. In Sect. 5 a short conclusion and discussion is given.

\section{The effective chiral confinement Lagrangian}

The main idea of the quark-chiral or Effective Chiral Confinement Lagrangian (ECCL), approach [33-37] is to take into account simultaneously pure chiral d.o.f. and quark d.o.f. connected first of all with confinement. The corresponding Lagrangian, called in [37] the Effective Chiral Confinement Lagrangian was derived in [33-37] in the following form

$L_{e f f}\left(M_{S}, \hat{\phi}\right)=-N_{c} \operatorname{tr} \log [i \hat{\partial}+\hat{m}+M \hat{U}]$.

where $\hat{U}=\exp \left(\hat{\phi} \gamma_{5}\right)$, and $M(x)$ is a confining kernel, which represents confinement of the quark with the mass $m_{i}$ (here $\hat{m}=\operatorname{diag}\left(m_{i}\right)$ ), in the common Wilson loop of the $q \bar{q}$ Green's function, as shown in Fig. 1. One can take $M(x)=\sigma|\mathbf{x}|$ as shown in Fig. 1, where it is convenient to take $\mathbf{x}=0$ at the midpoint of quark and antiquark. In what follows one encounters $M(x)$ at the vertex of the $q \bar{q}$ Green's function, which we associate with the minimal distance $x_{\min }=\lambda \cong 0.1$ fm equal to the vacuum correlation length in the QCD vacuum [33-35], and we introduce at those vertex new parameter $M(0)=M(\lambda)=\sigma \lambda=0.15 \mathrm{GeV}$ as the only parameter of our Lagrangian, besides $\sigma$ and current quark masses $m_{i}$. Note, that the behavior of $M(x)$ and the field correlator $\langle F(x) F(0)\rangle$ at small $x \ll \lambda$ is rather complicated, which precludes a simple zero limit $M(0)=0$. It is understandable that with magnetic field (m.f.) one defines $\hat{\partial} \rightarrow \hat{\partial}-i e \hat{A}^{(e)}$.

To proceed and to find the connection with the standard ECL [1-5], one defines the quark Green's function

$S_{0}=\frac{i}{\hat{\partial}+\hat{m}+M} \equiv i \Lambda$

and

$\eta=\hat{U}^{+} \Lambda(\hat{\partial}+\hat{m})(\hat{U}-1)=\eta_{\varphi}+\eta_{m}$

with

$\eta_{\varphi}=\hat{U}^{+} \Lambda \hat{\partial} \hat{U}, \quad \eta_{m}=\hat{U}^{+} \Lambda \hat{m}(\hat{U}-1)$,

so that $L_{e f f}$ acquires the form

$L_{e f f}=-N_{c} t r \log (1-\eta)$. 


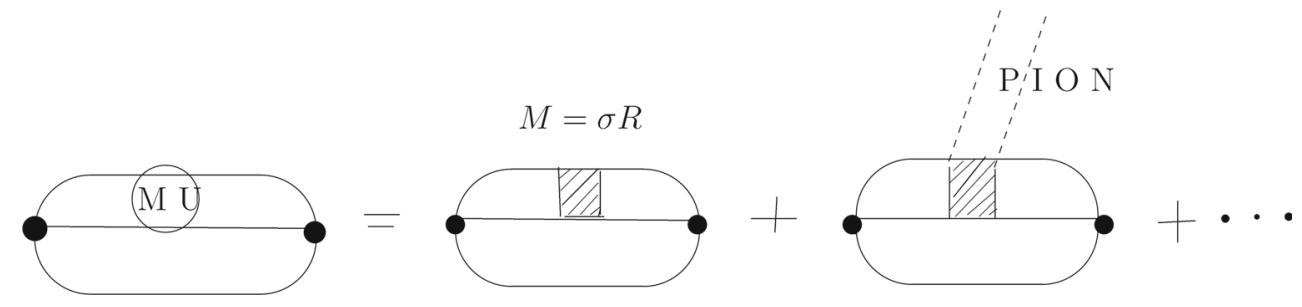

Fig. 1 The effective chiral confining kernel $M_{S} \hat{U}$ comprising confinement in $M_{S}$ and chiral mesons in $\hat{U}$

As a result the quadratic in $\hat{\partial} \hat{U}$ part of Lagrangian is

$L_{e f f}^{(2)}=\frac{1}{2} N_{c} \operatorname{tr}\left(\hat{U}^{+} \Lambda \hat{\partial} \hat{U}, \hat{U}^{+} \Lambda \hat{\partial} \hat{U}\right) \approx \frac{N_{c}}{2} \operatorname{tr}(\Lambda \hat{\partial} \hat{\varphi} \bar{\Lambda} \hat{\partial} \hat{\varphi})$,

with $\bar{\Lambda}=\hat{m}+M-\hat{\partial}, \hat{\varphi}=\frac{\varphi_{i} \lambda_{i}}{f_{i}}, f_{i}=f_{\pi}, f_{K}$ are numbers.

Here the sign $t r$ implies summation over flavor, Dirac and space-time coordinates.

To understand connection of $L_{\text {eff }}^{2)}$ in (10) with the standard ECL Lagrangian (4), one can express $\Lambda$ via the quadratic quark Green's function $G$ as

$\Lambda=(m+M-\hat{\partial}) G$.

As a result one obtains

$L_{\text {eff }}^{(2)}=\left\{\frac{\hat{f}_{\pi}^{2}}{4}\left(\partial_{\mu} U \partial_{\mu} U^{+}\right)\right\}$,

where the operator $\hat{f}_{\pi}^{2}$ can be written in terms of Hamiltonian eigenvalues.

For the latter one can exploit the path integral form, suggested in [42-45] and repeatedly used for all mesons and baryons. In terms of the n-th wave function $\varphi_{n}(r)$ of the quark $a$ and antiquark $b$ and eigenvalue $M_{n}$, as well as $\omega_{i}^{(n)}=\left\langle\sqrt{\mathbf{p}^{2}+m_{i}^{2}}\right\rangle_{n}, i=a, b$, the chiral decay constant is $[34,37,41-44]$

$$
\left(f_{a b}^{(n)}\right)^{2}=\frac{N_{c}\left(m_{a}+M(0)\right)\left(m_{b}+M(0)\right)}{2 \omega_{a}^{(n)} \omega_{b}^{(n)} M_{n} \xi_{n}} \varphi_{n}^{2}(0),
$$

where $\xi_{n}$ for light quarks is $\xi_{n}=1 / 2.34[44,45]$.

The good accuracy of (13), supported by comparison of calculations in [41-43,45] with experiment [46], allows to use the chiral quark theory of ECCL in the case of external m.f.

Note the difference between numbers $f_{i}, f_{k}$ and operators $\hat{f}_{i k}^{2}$, which contain derivatives $\hat{\partial}$ also in $G_{i}, G_{k}$ and hence can depend on external magnetic field. In absence of m.f. the expression (13) was used to calculate the decay contains of $f_{\pi}, f_{K}, f_{D}$ in the framework of the path integral Hamiltonian [47-49]. As a result one obtains the physical eigenvalues of $\hat{f}_{i k}^{2}$, which agree well with experimental values as shown in [42-45]. Finally, choosing these values as parameters $f_{i}$ in $\hat{\varphi}$, one accomplishes the standard ECL Lagrangian $L_{E C L}^{(2)}$, where $f_{i}$ serve as basic dimensional parameters of the theory.

In our case in the ECCL Lagrangian these parameters can be calculated in terms of basic QCD parameters: string tension $\sigma, \alpha_{s}$ and current quark masses $m_{i}$.

Using $M(0)=0.15 \mathrm{GeV}$ and $\sigma=0.18 \mathrm{GeV}^{2}$ one obtains in [45] for $\bar{f}_{\pi}=\sqrt{2} f_{\pi}, \bar{f}_{K}=\sqrt{2} f_{K}$ the following values

$\bar{f}_{\pi}=0.133 \mathrm{GeV}, \quad \bar{f}_{k}=0.165 \mathrm{GeV}$,

which should be compared with experimental values [46]

$$
\begin{aligned}
& \bar{f}_{\pi^{+}}^{(\exp )}=(130.7 \pm 0.1 \pm 0.36) \mathrm{MeV}, \\
& \bar{f}_{K^{+}}^{(\exp )}=(159.8 \pm 1.4 \pm 0.44) \mathrm{MeV} .
\end{aligned}
$$

In a similar way one computes $f_{\pi}, f_{K}$ for higher radial excitations, as it is shown in [45].

First of all one can check the GMOR relations [2] which follow from the ECCL in the second order $O\left(\eta^{2}\right)$.

$$
\begin{aligned}
L_{E C C L}^{(2)} & =N_{c} t r\left(\eta+\frac{\eta^{2}}{2}\right) \\
& =N_{c} t r\left\{\Lambda m \frac{\hat{\varphi}^{2}}{2}+\frac{1}{2} \Lambda \hat{\partial} \hat{\varphi} \bar{\Lambda} \hat{\partial} \hat{\varphi}-\frac{1}{2} \Lambda \hat{m} \hat{\varphi} \bar{\Lambda} \hat{m} \hat{\varphi}\right\}
\end{aligned}
$$

Taking into account that

$N_{c} \operatorname{tr} \Lambda \hat{m} \frac{\hat{\varphi}^{2}}{2}=\Delta_{a} m_{a} \varphi_{a b} \varphi_{b a}$

one obtains, neglecting $O\left(\mathrm{~m}^{2}\right)$ terms from the last term in (17),

$$
\begin{aligned}
f_{\pi^{0}}^{2} M_{\pi^{0}}^{2} & =\Delta_{1} m_{1}+\Delta_{2} m_{2} \\
f_{\pi^{+}}^{2} M_{\pi^{+}}^{2} & =f_{\pi^{-}}^{2} M_{\pi^{-}}^{2}=\Delta_{1} m_{1}+\Delta_{2} m_{2} \\
f_{K^{+}}^{2} M_{K^{+}}^{2} & =f_{K^{-}}^{2} M_{K^{-}}^{2}=\Delta_{1} m_{1}+\Delta_{3} m_{3} \\
f_{K^{0}}^{2} M_{K^{0}}^{2} & =\Delta_{2} m_{2}+\Delta_{3} m_{3} .
\end{aligned}
$$

Here $\Delta_{a}$ is the quark condensate, $\Delta_{a}=N_{c} \operatorname{tr} \Lambda_{a}$, where $1,2,3$ refer to $u, d, s$, and $m_{i}$ are pole quark masses, which are connected to the current quark masses in $\overline{M S}$ scheme (see [46] and [49]). One can see that (19)-(22) coincide with the standard GMOR relations [2]. 
Considering now the quark condensate $\Delta_{a}$ one has

$$
\begin{aligned}
\Delta_{a} & =N_{c}\left\langle\operatorname{tr} \Lambda_{a}\right\rangle \\
& =N_{c}\left\langle\operatorname{Tr}\left(\frac{1}{\hat{\partial}+m_{a}+M}\left(M+m_{a}-\hat{\partial}\right) \frac{1}{M+m_{a}-\hat{\partial}}\right)\right\rangle \\
& =N_{c}\left(m_{a}+M(0)\right) \operatorname{Tr}\left(\Lambda_{a} \bar{\Lambda}_{a}\right) \\
& =N_{c}\left(m_{a}+M(0)\right) \operatorname{Tr}\left(\Lambda_{a} \gamma_{5} \Lambda_{a} \gamma_{5}\right) .
\end{aligned}
$$

To proceed one can express $\Delta_{a}$ via the Green's function $G_{a a}(0)$, in [34,35], which was exploited in [38] in the case of nonzero m.f., so that $\Delta_{a}=N_{c}\left(m_{a}+M(0)\right) G_{a a}(0)$

$$
\begin{aligned}
G_{a b}(k)= & \int d^{4}(x-y) e^{i k(x-y)} \\
& \left.\times\left\langle\operatorname{Tr} \Lambda_{a}(x, y) \gamma_{5} \Lambda_{a}(y, x) \gamma_{5}\right)\right\rangle .
\end{aligned}
$$

Using the spectral decomposition of $G_{a b}(k)$,

$G_{a b}(k)=\sum_{n=0}^{\infty} \frac{c_{n}}{k^{2}+\bar{M}_{n}^{2}}$,

one finds

$\Delta_{a}=N_{c}\left(M(0)+m_{a}\right) \sum_{n=0}^{\infty} \frac{\varphi_{n}^{2}(0)}{M_{n}}$.

Here $\varphi_{n}(r), M_{n}$ are eigenfunction and eigenvalue of the Green's function $G_{a b}$ and the corresponding Hamiltonian, found in [48-50], which correspond to $J^{P C}=0^{-+}$and do not contain chiral d.o.f. In a similar way from $G_{a b}(k)=$ $G_{a b}(0)+\frac{k^{2} f_{\pi}^{2}}{N_{c}}+\cdots$ one obtains as in $[34,35]$ for $f_{a b}$.

$f_{a b}^{2}=N_{c}\left(M(0)+m_{a}\right)\left(M(0)+m_{b}\right) \sum_{n=0}^{\infty} \frac{\left|\varphi_{n}(0)\right|^{2}}{M_{n}^{3}}$

Note, that forms (13) and (27) are equivalent, since for the PS states $\varphi_{n} 2 \omega_{a}^{2} \xi_{n} \approx M_{n}^{2}, M_{n} \approx 2 \omega_{a}(n)$.

In what follows we shall mostly use the forms (26), (27). To proceed one must detalize the Hamiltonian technic, which produces $M_{n}, \varphi_{n}(0)$ to prepare for the inclusion of m.f. in this Hamiltonian.

Note, that confinement is separated in $\Lambda_{a}=\frac{1}{\hat{\partial}+m+M(x)}$ as the interaction term $M(x)=\sigma|\mathbf{x}|$, which implies the use of the instantaneous interaction $V_{q \bar{q}}(\mathbf{x}-\mathbf{y})=M_{q}(\mathbf{x})+M_{\bar{q}}(\mathbf{x})$, between $q$ and $\bar{q}$. This form was useful above to introduce together with confinement the chiral d.o.f. as $M(\mathbf{x}) \rightarrow$ $M(\mathbf{x}) U(x)$.

To calculate effects of confinement and all spin corrections in our case of $G_{a b}(x, y)$, where chiral d.o.f. do not participate, it is more convenient to go back to original QCD Green's function with confinement, as it is done in [44,45].

Now using the path integral representation in the Euclidean space-time with the proper time $s_{i}=\frac{T_{4}}{2 \omega_{i}}, T_{4}=x_{4}-y_{4}$, one has as in $[44,45]$

$$
\begin{aligned}
I_{a b} & \equiv\left(\frac{1}{\left(m_{a}^{2}-\hat{D}_{a}^{2}\right)\left(m_{b}^{2}-\hat{D}_{b}^{2}\right)}\right)_{x y} \\
& =\frac{T_{4}}{8 \pi} \int_{0}^{\infty} \frac{d \omega_{a}}{\omega_{a}^{3 / 2}} \int_{0}^{\infty} \frac{d \omega_{b}}{\omega_{b}^{3 / 2}}\left(D^{3} z_{a}\right)_{\mathbf{x y}}\left(D^{3} z_{b}\right)_{\mathbf{x y}} e^{-A}
\end{aligned}
$$

where

$A=K_{a}\left(\omega_{a}\right)+K_{b}\left(\omega_{b}\right)+\int_{0}^{T_{4}} d t_{E} V_{0}\left(r\left(t_{E}\right)\right)$

and $V_{0}(r)$ is the result of the instantaneous interaction from the Wilson loop

$V_{0}(r)=V_{\text {conf }}(r)+V_{O G E}(r)+\Delta V$,

where $\Delta V$ includes spin-depend part, and

$K_{i}=\frac{m_{i}^{2}+\omega_{i}^{2}}{2 \omega_{i}} T_{4}+\int_{0}^{4} d t_{E} \frac{\omega_{i}}{2}\left(\frac{d \mathbf{z}^{(i)}}{d t_{E}}\right)^{2}, \quad i=a, b$.

From (28)-(31) one obtains in a standard way the Hamiltonian

$I_{a b}=\frac{T_{4}}{8 \pi} \int_{0}^{\infty} \frac{d \omega_{a}}{\omega_{a}^{3 / 2}} \int_{0}^{\infty} \frac{d \omega_{b}}{\omega_{b}^{3 / 2}}\left\langle\mathbf{x}, \mathbf{x}\left|e^{-H\left(\omega_{a}, \omega_{b}, \mathbf{p}_{a}, \mathbf{p}_{b}\right) T_{4}}\right| \mathbf{y}, \mathbf{y}\right\rangle$,

and

$$
\begin{aligned}
H= & \sum_{i=a, b} \frac{\left(\mathbf{p}^{(i)}\right)^{2}+m_{i}^{2}+\omega_{i}^{2}}{2 \omega_{i}}+V_{0}(r)=\frac{\mathbf{P}^{2}}{2\left(\omega_{1}+\omega_{2}\right)} \\
& +\frac{\mathbf{p}^{2}}{2 \tilde{\omega}^{2}}+V_{0}(r) \equiv \frac{\mathbf{P}^{2}}{\left(2\left(\omega_{1}+\omega_{2}\right)\right.}+h
\end{aligned}
$$

where $\tilde{\omega}=\frac{\omega_{a} \omega_{b}}{\omega_{a}+\omega_{b}}$.

As a result the matrix element $\left\langle\left|e^{-H T_{4}}\right|\right\rangle$ in (32) can be written as

$\int d^{3}(\mathbf{x}-\mathbf{y})\left\langle\mathbf{x}, \mathbf{x}\left|e^{-H T_{4}}\right| \mathbf{y}, \mathbf{y}\right\rangle=\sum_{n} \varphi_{n}^{2}(0) e^{-M_{n}\left(\omega_{a}, \omega_{b}\right) T_{4}}$,

where $\varphi_{n}, M_{n}$ are eigenvalues of $h \equiv \frac{\mathbf{p}^{2}}{2 \tilde{\omega}}+V_{0}(r), h \varphi_{n}=$ $M_{n} \varphi_{n}$.

In the limit $T_{4} \rightarrow \infty$ one can use the stationary point analysis of the integral $\int I_{a b} d^{3}(\mathbf{x}-\mathbf{y})=\int_{0}^{\infty} \frac{d \omega_{a}}{\omega_{a}^{3 / 2}} \int_{0}^{\infty} \frac{d \omega_{b}}{\omega_{b}^{3 / 2}} \varphi_{n}^{2}(0)$ $e^{-M_{n}\left(\omega_{a}, \omega_{b}\right) T_{4}}$, which finally yields the stationary values $\omega_{a}^{(0)}, \omega_{b}^{(0)}$ from the condition $\left.\frac{\partial M_{n}}{\partial \omega_{i}}\right|_{\omega_{i}=\omega_{0}^{(0)}}=0, i=a, b$, and the final physical eigenvalues $M_{n}^{(0)}=M_{n}\left(\omega_{i}^{(0)}\right)$ and eigenfunctions $\varphi_{n}^{(0)}=\varphi_{n}\left(\omega_{i}^{(0)}, \mathbf{r}\right)$.

As it was shown in $[34,35]$ the resulting eigenvalues of the (nonchiral) PS states with confinement, color Coulomb and spin-spin interactions taken into account, are $M_{0}=0.4$ 
$\mathrm{GeV}, M_{1}=1.35 \mathrm{GeV}$ and $M_{2}=1.85$, while $\left|\varphi_{n}(0)\right|^{2}=$ $\frac{\omega_{n}^{(0)}}{4 \pi}\left(\sigma+\frac{4}{3} \alpha_{s}\left\langle\frac{1}{r^{2}}\right\rangle\right)$.

Finally, using (27) one obtains the value of $f_{\pi^{0}}^{2} \cong f_{u \bar{u}}^{2} \cong$ $f_{d \bar{d}}^{2}=94 \mathrm{MeV}$, where the first 3 states of the fast converging series in (27) are taken into account. This value agrees well with (14), obtained in [45] in a different way.

For $\Delta_{a}$ the same values of $M_{n},\left|\varphi_{n}(0)\right|^{2}$ can be taken but the series is formally diverging and must be renormalized. We shall not touch this point below, since we shall need the difference $\frac{\Delta_{a}\left(e_{a} B\right)-\Delta_{a}(0)}{\Delta_{a}(0)}$, where only few first terms contribute.

In the next section we generalize this derivation imposing m.f. on our $q \bar{q}$ system.

\section{The $q \bar{q}$ system in magnetic field}

In this case the Hamiltonian can be written as $[51,52]$

$$
\begin{aligned}
H= & \sum_{i=a, b}\left(\frac{\left(\mathbf{p}_{i}-e_{q} \bar{A}^{(e)}\right)^{2}}{2 \omega_{i}}+\frac{m_{i}^{2}+\omega_{i}^{2}}{2 \omega_{i}}-\frac{e_{i} \boldsymbol{\sigma}_{i} \mathbf{B}}{2 \omega_{i}}\right) \\
& +V_{0}(R)
\end{aligned}
$$

and for the neutral systems the relative motion Hamiltonian is

$$
\begin{gathered}
h=\frac{1}{2 \tilde{\omega}}\left(-\frac{d^{2}}{d \mathbf{r}^{2}}+\left(\frac{e_{q}(\mathbf{r} \times \mathbf{B})}{2}\right)^{2}\right) \\
+V_{0}(r)+\sum_{i=a, b} \frac{e_{i} \boldsymbol{\sigma}_{i} \mathbf{B}}{2 \omega_{i}}
\end{gathered}
$$

An analytic answer for energy eigenvalue and eigenfunctions can be obtained with $O(5 \%)$ accuracy replacing the linear confinement by the quadratic form with the subsequent stationary point analysis of coefficients

$V_{\text {conf }}^{(\text {lin })}=\sigma r \rightarrow \frac{\sigma}{2}\left(r^{2} \gamma+\frac{1}{\gamma}\right),\left.\frac{\partial M}{\partial \gamma}\right|_{\gamma=\gamma_{0}}=0$,

which yields the final result

$M_{\mathbf{n}}=\varepsilon_{\mathbf{n}}+\frac{m_{a}^{2}+\omega_{a}^{2}-e_{a} \mathbf{B} \boldsymbol{\sigma}_{a}}{2 \omega_{a}}+\frac{m_{b}^{2}+\omega_{b}^{2}-e_{b} \mathbf{B} \boldsymbol{\sigma}_{b}}{2 \omega_{b}}$.

Here $\varepsilon_{\mathbf{n}}$ is

$$
\begin{aligned}
\varepsilon_{\mathbf{n}}= & \frac{1}{2 \tilde{\omega}}\left(\sqrt{e_{q}^{2} B^{2}+\sigma^{2} c}\left(2 n_{\perp}+1\right)+\sqrt{\sigma^{2} c}\left(n_{3}+\frac{1}{2}\right)\right) \\
& +\frac{\gamma \sigma}{2}
\end{aligned}
$$

where $n_{\perp}, n_{z}$ are oscillator quantum numbers, and $c=\frac{4 \tilde{\omega}}{\gamma \sigma}$, while $\gamma \rightarrow \gamma_{0}$ is defined from the minimum of $M_{\mathbf{n}}(\gamma)$.

The index $\mathbf{n}$ here denotes $n_{\perp}, n_{3}$ and two possible relative orientation of $\sigma_{a}, \sigma_{b}$ with respect to $\mathbf{B},(+-)$ and (-+).
The most important role in what follows is played by the factor $\left|\varphi_{n}(0)\right|^{2}$ in (26), (27), which is easily calculated in the oscillator potential approximation to be

$$
\begin{aligned}
\left|\varphi_{n}(0)\right|^{2} & =\frac{1}{\pi^{3 / 2} r_{\perp}^{2} r_{3}}, \quad r_{\perp}^{2}=2\left(\left(e_{q} B\right)^{2}+\sigma^{2} c\right)^{-1 / 2}, \\
r_{3} & =\left(\frac{\sigma^{2} c}{4}\right)^{-1 / 4} .
\end{aligned}
$$

Defining $c=c_{+-}, c_{-+}$by stationary point analysis one find that

$c_{+-}(B) \approx 1, \quad c_{-+}(B) \cong\left(1+\frac{8 e_{q} B}{\sigma}\right)^{2 / 3}$

and as a result one has [38]

$$
\begin{aligned}
& \left|\psi_{n_{\perp}=0, n_{3}}^{(+-)}(0)\right|^{2} \cong \frac{\sqrt{\sigma} \sqrt{e_{q}^{2} B^{2}+\sigma^{2}}}{(2 \pi)^{3 / 2}} \\
& \left|\psi_{n_{\perp}=0, n_{3}}^{(-+)}(0)\right|^{2}=\left(\sigma^{2} c_{-+}\right)^{3 / 4} \sqrt{1+\left(\frac{e_{q} B}{\sigma}\right)^{2} \frac{1}{c_{-+}}} .
\end{aligned}
$$

It is interesting, that the energy $M_{\mathbf{n}}$ of the lowest level with $n_{\perp}=n_{3}=0$ is very different in the $(+-)$ and $(-+)$ cases. Indeed $M_{0}^{(+-)}$is slowly changing and tends to the constant limit, while $M_{0}^{(-+)}$, where the cancellation of the terms $e_{i} \boldsymbol{\sigma}_{i} \mathbf{B}$ does not take place, is growing with $e B$ :

$M_{0}^{(-+)}(a, a) \approx 2 \sqrt{2\left|e_{a} B\right|}, \quad M_{0}^{(+-)} \cong$ const.

We now can write expressions for $(\Sigma, F, M)$ in m.f. in a general form, generalizing Eqs. (26), (27) since now the sum over $n$ is going over $n_{\perp}, n_{3}$ and $(+-),(-+)$, so that one has $[39,40]^{1}$

$$
\begin{aligned}
& f_{\pi^{0}}^{2}\left(e_{q} B\right) \\
& \quad=N_{c}\left(M(0)+m_{i}\right)^{2} \sum_{n_{\perp}, n_{3}}\left(\frac{\frac{1}{2}\left|\psi_{n i}^{(+-)}(0)\right|^{2}}{\left(M_{n i}^{(+-)}\right)^{3}}+\frac{\frac{1}{2}\left|\psi_{n i}^{(-+)}(0)\right|^{2}}{\left(M_{n i}^{(-+)}\right)^{3}}\right),
\end{aligned}
$$

where $i=u, d$. In the same way $\Delta_{a}$ acquires the form [38, 39]

$$
\begin{aligned}
\Delta_{i} & =N_{c}\left|\left\langle\bar{q}_{i} q_{i}\right\rangle\right| \\
& =N_{c}\left(M(0)+m_{i}\right) \sum_{n_{\perp}, n_{3}}\left(\frac{\frac{1}{2}\left|\psi_{n i}^{(+-)}(0)\right|^{2}}{M_{n i}^{(+-)}}+\frac{\frac{1}{2}\left|\psi_{n i}^{(-+)}(0)\right|^{2}}{M_{n i}^{(-+)}}\right) .
\end{aligned}
$$

Finally, it was found in [39], that GMOR relations [2] for neutral mesons are conserved in m.f., so that we can write

$m_{\pi^{0}}^{2} f_{\pi^{0}}^{2}=\frac{\bar{m} M(0)}{M(0)+\bar{m}}|\langle\bar{u} u\rangle+\langle\bar{d} d\rangle|, \quad \bar{m}=\frac{m_{u}+m_{d}}{2}$

\footnotetext{
${ }^{1}$ Note an erroneous dependence of $M^{(+-)}(e B)$ on m.f. in [40] and as a result a fast growth of $f_{\pi^{0}}(e B)$. In what follows we shall not use these results.
} 
and one can use GMOR relations separately for $u$ and $d$ flavors. In the next section we shall find analytically and numerically the behavior of $\Delta_{i}, f_{\pi^{0}}$ and $m_{\pi^{0}}$ in m.f.

\section{Behavior of chiral observables $(\Sigma, f, M)$ in m.f}

One can define explicitly the behavior of $\Sigma, f, M$ in m.f. introducing the relative growth coefficients

$$
\begin{aligned}
& \frac{\Delta_{a}\left(e_{a} B\right)-\Delta_{a}(0)}{\Delta(0)} \equiv \Delta \Sigma_{a}, a=u, d . \\
& \frac{f_{\pi^{0}}^{2}\left(e_{a} B\right)}{f_{\pi^{0}}^{2}(0)}=K_{f}^{(a)}(B) \\
& \frac{m_{\pi^{0}}^{2}(B)}{m_{\pi^{0}}^{2}(0)}=K_{\pi^{0}}(B) .
\end{aligned}
$$

One can notice in (45), (46), that masses $M_{n i}$ in the denominator strongly increase, when one is going from $n_{\perp}, n_{3}=0,0$ to higher values and there occurs a significant compensation of higher $n_{\perp}, n_{3}$ terms in the difference $\Delta_{a}(B)-\Delta_{a}(0)$ etc. Therefore the main contribution to the coefficients $\Delta \Sigma_{a}, K_{f}, K_{\pi}$ is given by the lowest term with $n_{\perp}, n_{3}=0,0$, which we retain and compare to the lattice data.

For $\Delta \Sigma_{a}(B)$ one obtains from (46), (42), (43)

$$
\begin{aligned}
& \Delta \Sigma_{a}=\frac{1}{2}\left\{\sqrt{1+\left(\frac{e_{a} B}{\sigma}\right)^{2}}+\sqrt{1+\left(\frac{e_{a} B}{\sigma}\right)^{2} \frac{1}{c_{-+}^{(a)}}}-2\right\}, \\
& a=u, d .
\end{aligned}
$$

For $K_{f}^{(a)}\left(e_{a} B\right)$ one can use (44), (45) and neglect the terms $(-+)$ in (45), since $M_{n}^{(-+)}$is fast growing at large $e B$. As a result one obtains a simple estimate

$K_{f}^{(a)}\left(e_{a} B\right) \cong \sqrt{1+\left(\frac{e_{a} B}{\sigma}\right)^{2}}, \quad e_{a} B \gtrsim \sigma$

Finally for $m_{\pi^{0}}^{2}$ one has form (47)

$\frac{m_{\pi^{0}}^{2}\left(e_{a} B\right)}{m_{\pi^{0}}^{2}(0)}=\frac{\Delta_{u}\left(e_{u} B\right)}{f_{\pi^{0}}^{2}\left(e_{u} B\right)} \frac{f_{\pi^{0}}^{2}(0)}{\Delta_{u}(0)}=\frac{1+\Delta \Sigma_{a}}{K_{f}^{(a)}\left(e_{a} B\right)}$.

We start with the quark condensate, Eq. (51) and define two functions in analogy with [25]:

$$
\begin{aligned}
\frac{1}{2}\left(\Delta \Sigma_{u}+\Delta \Sigma_{d}\right) & \equiv K_{+}(e B) \\
\Delta \Sigma_{u}-\Delta \Sigma_{d} & \equiv K_{-}(e B)
\end{aligned}
$$

In Figs. 2, 3 we show both functions (54), (55) computed with the help of (51) in comparison with the chiral perturbation theory result (1), and with the lattice calculated ratios in [25]. One can see a very good agreement of our result with

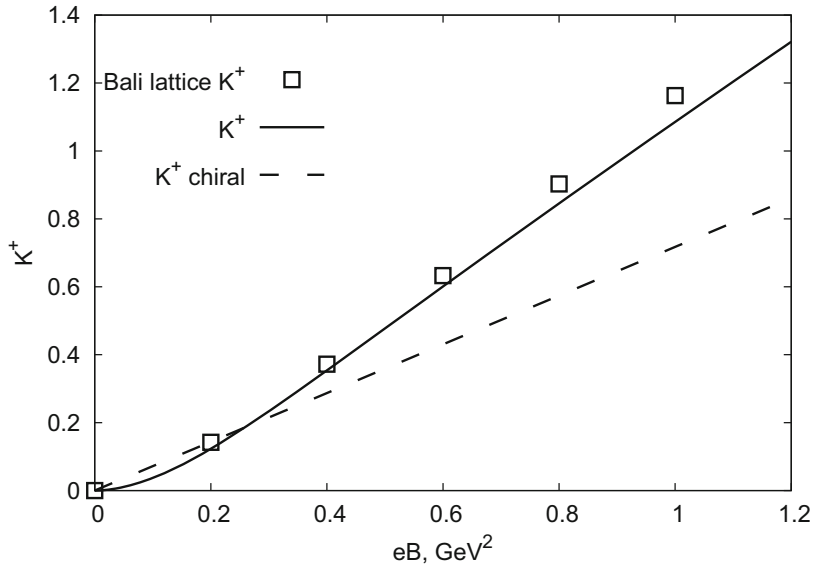

Fig. 2 The $K_{+}(e B$ calculated with ECL (solid line) in comparison with the lattice data from [25] and chiral perturbation theory (dashed line)

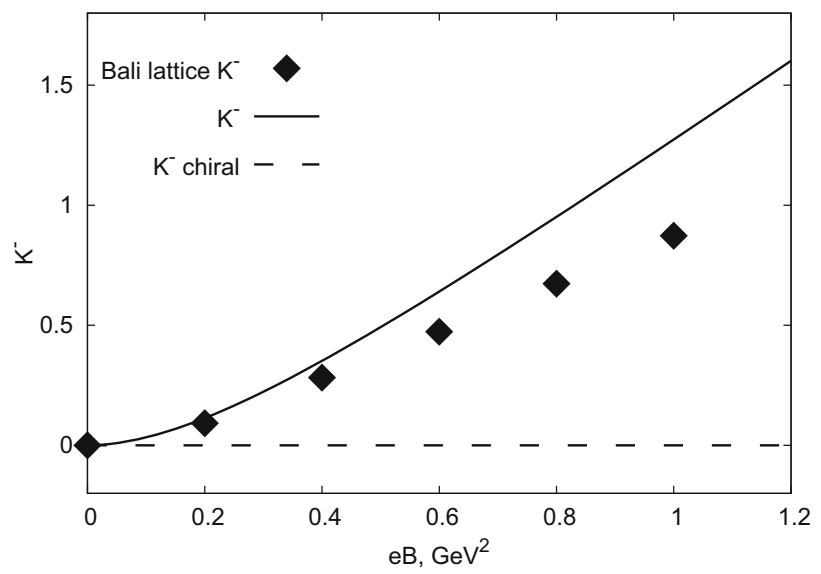

Fig. 3 The $K_{-}(e B$ calculated with ECL (solid line) in comparison with the lattice data from [25] and chiral perturbation theory (dashed line)

lattice data for all measured $e B \leq 1.1 \mathrm{GeV}^{2}$. One notice two important distinctions of our and lattice results with the ECL results

1. $\Delta \Sigma_{q}$ is roughly proportional to $\left|e_{q} B\right|$ at large $e_{q} B \gtrsim \sigma$, and hence $\Delta \Sigma_{u} \approx 2 \Delta \Sigma_{d}$, while in (1) $\Delta \Sigma_{u}=\Delta \Sigma_{d}$, so that in ChPT $K_{-}(e B) \equiv 0$.

2. The asymptotic linear behavior of $\frac{1}{2}\left(\Delta \Sigma_{u}+\Delta \Sigma_{d}\right)$ from (51), (54) is $\left(\frac{e B}{4 \sigma}\right)=\frac{1.4 e B}{1 \mathrm{GeV}^{2}}$, and is given by the $(+-)$ states, which agrees very well numerically with lattice data [25], while in ChPT $K_{+} \cong \frac{0.5 e B}{1 \mathrm{GeV}^{2}}$.

We now turn to the case of $f_{\pi^{0}}^{2}$ and display in Fig. 4 our result (53) in comparison with the ChPT result, Eq. (2). One can see different behavior of these two results. In our case the asymptotics is given in (52), 


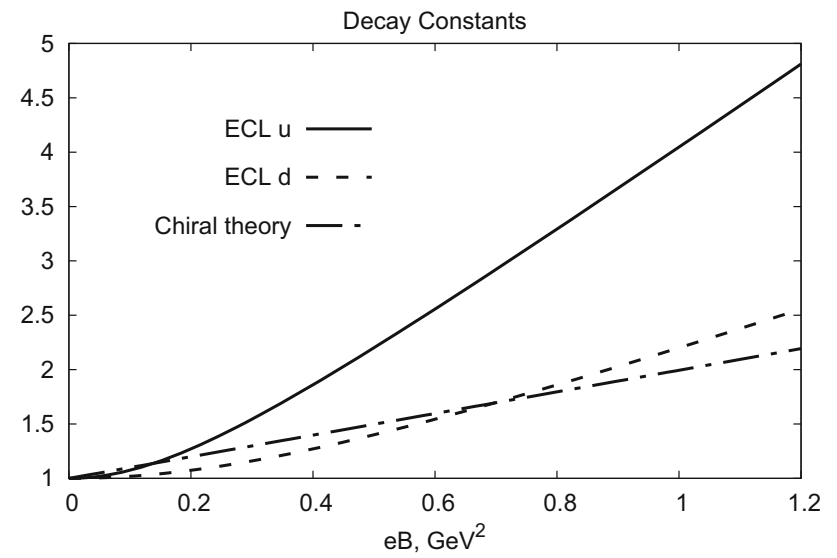

Fig. 4 The decay constants $\frac{f_{\pi^{0}}^{2}(e B)}{f_{\pi^{0}}^{2}(0)}$ for $u \bar{u}$ (solid) and $d \bar{d}$ (dashed) quark constituents calculated with ECL in comparison with the standard chiral perturbation theory

$K_{f}^{(a)} \cong \frac{e_{a} B}{\sigma} \approx(3.7,1.85) \frac{e B}{1 \mathrm{GeV}^{2}}$, for $(u, d)$ while in $\mathrm{ChPT}$ result (2), this ratio is $\frac{\ln 2 e B}{8 \pi^{2} F_{\pi}^{2}} \cong 0.25 \frac{e B}{1 \mathrm{Gev}^{2}}$.

We finally come to the $\pi^{0}$ mass problem, which according to (53) can be written as

$\frac{m_{\pi^{0}}^{2}\left(e_{q} B\right)}{m_{\pi^{0}}^{2}(0)}=\frac{1+A}{2}$

with

$A=\left[\frac{1+\left(\frac{e_{q} B}{\sigma}\right)^{2} \frac{1}{c_{-+}}}{1+\left(\frac{e_{a} B}{\sigma}\right)^{2}}\right]^{1 / 2}$.

The resulting curves for $q=u, d$ a shown in Fig. 5,6 together with the lattice data from [31,32] and the ChPT result Eq. (3) from [10]. One can see a reasonable agreement of our result with lattice data [31,32] and again a strong disagreement with Eq. (3).

\section{Results and discussion}

Our results for $\Sigma, f, M$ are shown in Figs. 2, 3, 4, 5 and 6 in comparison with lattice data and results of ChPT, Eq. (1), (2), (3). One can see a good agreement of our results in Figs. 2, 3, 5, 6 with lattice data from [25] and [31,33] respectively, while in all Figs. 2, 3, 4, 5 and 6 an apparent disagreement with the ChPT, Eqs. (1), (2), (3). One should stress that our equations for $(\Sigma, f, M)$ do not contain any fitting parameters and depend only on $\frac{e_{q} B}{\sigma}$, where $\sigma=0.16 \mathrm{GeV}^{2}$ is the standard QCD string tension.

It is understandable, that in our approach the basic effect is from the quark d.o.f (as it seen in the coefficients $\frac{e B}{\sigma}$ ), and the agreement with independent lattice data shows that

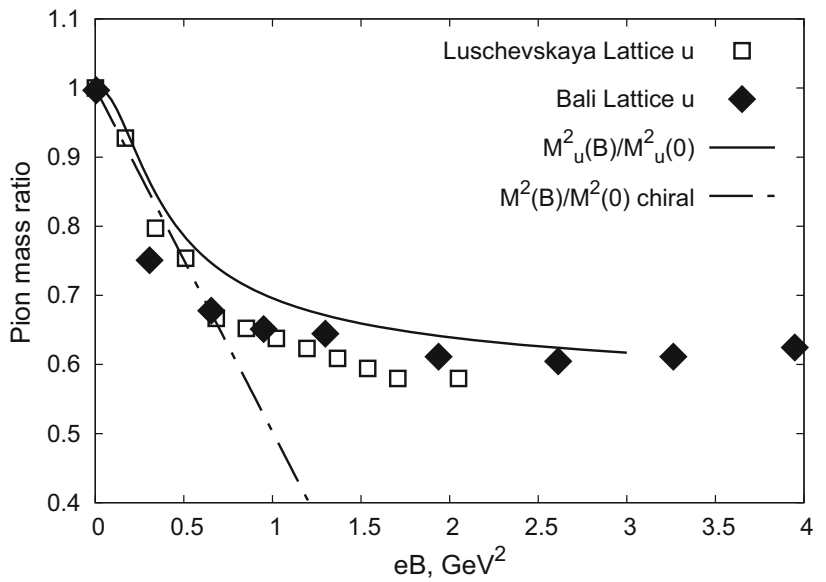

Fig. 5 The pion mass ratio $\frac{m_{\pi^{0}}^{2}(e B)}{e_{\pi^{0}}^{2}(0)}$ with $u \bar{u}$ quark constituents calculated with ECL (solid line) in comparison with the lattice data from $[31,32]$ (dots) and with the standard chiral perturbation theory prediction from [10]

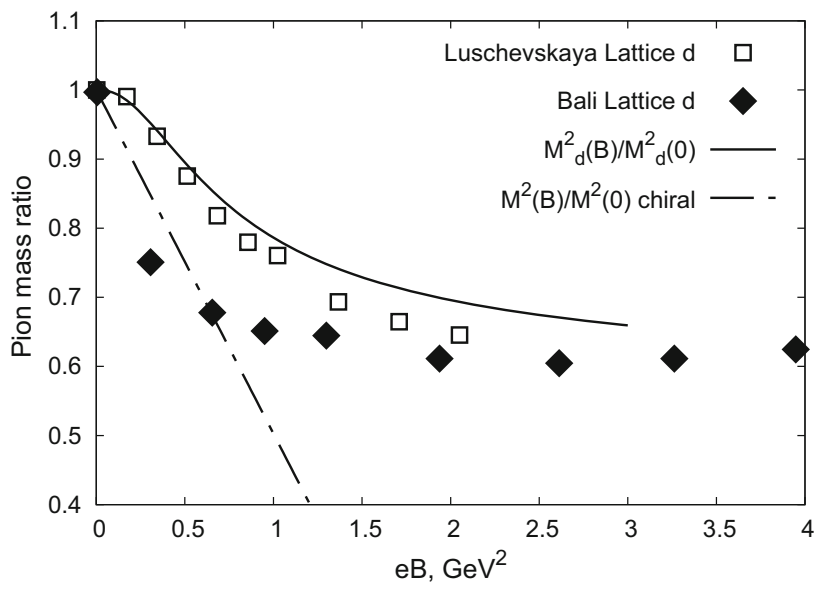

Fig. 6 The pion mass ratio $\frac{m_{\pi^{0}}^{2}(e B)}{e_{\pi^{0}}^{2}(0)}$ with $d \bar{d}$ quark constituents calculated with ECL (solid line) in comparison with the lattice data from $[31,32]$ (dots) and with the standard chiral perturbation theory prediction from [10]

this effect is properly taken into account. One of the points is then: where is the contribution from purely chiral d.o.f.? This point is especially aggravated, when one compares the coefficient $K_{-}(e B),(55)$ in Fig. 3, which according to ChPT should be identically zero, while in our and lattice approaches it is essentially nonzero and is of the same order, as the total ChPT correction to $\Delta(e B)$.

In our derivation of $\Delta_{a}$ and $\Delta \Sigma_{a}$, Eqs. (26), (48) the chiral corrections are absent and they appear in ECCL only in higher orders, in the terms $O\left(\Lambda(\hat{\partial} \varphi)^{n}\right), n>2$. The same happens in the case of $f_{\pi^{0}}, M_{\pi^{0}}$, where to the lowest order the terms $\left(\partial_{\mu} \pi^{ \pm}\right)^{2}$ are absent.

As a general feature, one should stress, that the ECCL is nonlocal in chiral variables and e.g. the pion propagator 
is replaced by the $q \bar{q}$ Green's function with pion quantum numbers, therefore the purely local chiral magnetic effects can be expected only for very small momenta and m.f.

We have not considered above the charged mesons $\pi^{ \pm}, K^{ \pm}$, where GMOR relations are violated in the lowest order,as it was found in [39]. The corresponding mass evolution for $M_{\pi^{ \pm}}$is given in [41].

One can compare our and lattice results with other approaches beyond ChPT. In [25] the lattice results for $K_{+}$ have been compared to the PNJL model of [53], showing a reasonable agreement for $e B \lesssim 0.3 \mathrm{GeV}$, and deviation from lattice data for larger $e B$. A better agreement of $K_{+}$with lattice data in the whole interval $e B \leq 1 \mathrm{GeV}^{2}$ was found in [54], in the framework of the NJL model with a Gaussian formfactor. The $\pi^{0}$ mass in the NJL model, $M_{\pi^{0}}(e B)$, was found numerically in [55-57]. One can see in Fig. 2 of [57] almost the same slope, as in our Figs. 5, 6, slightly different (within 15\%) from the lattice data of [33] and within errors with data of [31]. A good agreement of [54] Fig. 4, with our data can be found for $f_{\pi^{0}}^{2}(e B)$ in Fig. 4. These coincidences support the main outcome of our paper, that the quark d.o.f., taken into account also within the NJL model, play the most important role in the impact of m.f.

It is interesting to identify the explicit mechanism, which provides the linear growth of $\Sigma_{a}(e B)$, and $f_{\pi^{0}}(e B)$ with increasing $e B$. Indeed, looking at Eqs. (42), (43) and (45), (46) one can notice, that the main effect of increase comes from the factor $|\varphi(0)|^{2} \sim \sqrt{1+\left(\frac{e_{q} B}{\sigma}\right)^{2}}$, which is a familiar effect of the $q \bar{q}$ attraction at small distances in m.f., called in [58] the "magnetic focusing effect". This effect is present both in relativistic and nonrelativistic systems. One can notice that it is specially important in the spin-spin interactions, providing collapse of the $q \bar{q}$ system in the lowest local approximation of $h f$ interaction in m.f. This point was treated in [52,59], where it was shown that an effective smearing is necessary for spin-spin forces in m.f., which prevents collapse and satisfies the positivity conditions for eigenvalues. This kind of treatment is also assumed in our case.

It is possible, that the agreement of our results with the corresponding data from $[54,57]$ is due to the same simple magnetic focusing effect, discussed above.

Having found that purely chiral ECL Lagrangian, not containing quark d.o.f., does not ensure the correct behavior of the physical system under the influence of m.f., one may ask, what happens to the so-called chiral magnetic effects in similar purely chiral Lagrangians? This question requires a detailed analysis and a possible extension of these purely chiral Lagrangians to the quark-chiral form, as it is done in the extension of $L_{E C L}$ to $L_{E C C L}$ in [37].

Acknowledgements This work was supported by the Russian Science Foundation Grant 16-12-10414.
Open Access This article is distributed under the terms of the Creative Commons Attribution 4.0 International License (http://creativecomm ons.org/licenses/by/4.0/), which permits unrestricted use, distribution, and reproduction in any medium, provided you give appropriate credit to the original author(s) and the source, provide a link to the Creative Commons license, and indicate if changes were made.

Funded by $\mathrm{SCOAP}^{3}$.

\section{References}

1. S.L. Glashow, S. Weinberg, Phys. Rev. Lett. 20, 224 (1968)

2. M. Gell-Mann, R.L. Oakes, B. Renner, Phys. Rev. 175, 2195 (1968)

3. S.O. Okubo, Phys. Rev. 188, 2295 (1969)

4. S. Weinberg, Phys. A 96, 327 (1979)

5. J. Gasser, H. Leutwyler, Ann. Phys. 158, 142 (1984)

6. J. Bijnens, G. Colangelo, G. Ecker, Ann. Phys. 280, 100 (2000). arxiv:hep-ph/9907333

7. H. Georgi, Ann. Rev. Nucl. Part. Sci. 43, 209 (1993)

8. S. Weiberg, Int. J. Mod. Phys. A 31, 1630007 (2016)

9. C.P. Burgess, Annu. Rev. Nucl. Part. Sci. 57, 329 (2007). arxiv:hep-th/0701053

10. I.A. Shushpanov, A.V. Smilga, Phys. Lett. B 402, 351 (1997). arxiv:hep-ph/9703201

11. N.O. Agasian, I.A. Shushpanov, Phys. Lett. B 472, 143 (2000). arxiv:hep-ph/9911254

12. T.D. Cohen, D.A. Mc Gady, E.S. Werbos, Phys. Rev. C76, 055201 (2007). arXiv:0706.3208

13. E.S. Werbos, Phys. Rev. C 77, 065202 (2008). arXiv:0711.2635

14. N.O. Agasian, Phys. Lett. B 488, 39 (2000). arxiv:hep-ph/0005300

15. N.O. Agasian, Phys. Atom. Nucl. 64, 554 (2001). arxiv:hep-ph/0112341

16. N.O. Agasian, I.A. Shushpanov, JHEP 0110, 006 (2001). arxiv:hep-ph/10107128

17. J.O. Andersen, JHEP 1210, 005 (2012). arXiv: 1205.6978

18. S.P. Klevansky, R.H. Lemmer, Phys. Rev. D 39, 3478 (1989)

19. V.P. Gusynin, V.A. Miransky, I.A. Shovkovy, Phys. Lett. B 349, 477 (1995). arxiv:hep-ph/9412257

20. AYu. Babansky, E.V. Gorbar, G.V. Shchepanyuk, Phys. Lett. B 419, 272 (1998). arxiv:hep-th/9705218

21. D.T. Son, M.A. Stephanov, Phys. Rev. D 77, 014021 (2008). arXiv: 0710.1084

22. M. D'Elia, F. Negro, Phys. Rev. D 83, 114028 (2011). arXiv: 1103.2080

23. M. D'Elia, S. Mukherjee, F. Sanfilippo, Phys. Rev. D 82, 051501 (2010). arXiv:1005.5365

24. E.-M. Ilgenfritz, M. Kalinowski, M. Muller-Preussker, B. Petersson, A. Scheiber, Phys. Rev. D 85, 114504 (2012). arXiv: 1203.3360

25. G.S. Bali, F. Bruckmann, G. Endrodi, Phys. Rev. D 86, 071502 (2012). arXiv:1206.4205

26. G.S. Bali, F. Bruckmann, G. Endrodi, S.D. Katz, A. Schafer, JHEP 08, 177 (2014). arXiv: 1406.0269

27. C. Bonati, M. D’Elia, M. Mariti, F. Negro, Phys. Rev. D 89, 114502 (2014)

28. C. Bonati, M. D'Elia, A. Ricci, Phys. Rev. D 92, 054014 (2015)

29. E.V. Luschevskaya, O.E. Solovjeva, O.V. Teryaev, Phys. Lett. B 761, 393 (2016)

30. E.V. Luschevskaya, O.E. Kochetkov, O.V. Larina, O.V. Teryaev, Nucl. Phys. B 884, 1 (2014)

31. E.V. Luschevskaya, O.E. Solovjeva, O.E. Kochetkov, O.V. Teryaev, Nucl. Phys. B 898, 627 (2015)

32. G.S. Bali, B.B. Brandt, G. Endrodi, B. Glaessle, Phys. Rev. D 97, 034505 (2018)

33. YuA Simonov, Phys. Rev. D 65, 094018 (2002). arxiv:hep-ph/0201170 
34. Yu A. Simonov, Phys. At. Nucl. 67, 846 (2004). arxiv:hep-ph/0302090

35. Yu A. Simonov, Phys. At. Nucl. 67, 1027 (2004). arxiv:hep-ph/0305281

36. S.M. Fedorov, Yu A. Simonov, JETP Lett. 78, 57 (2003). arxiv:hep-ph/0306216

37. Yu A. Simonov, Int. Mod. Phys. A 31, 165016 (2016). arXiv:1509.06930

38. Yu A. Simonov, JHEP 1401, 118 (2014). arXiv: 1212.3118

39. V.D. Orlovsky, YuA Simonov, JHEP 1309, 136 (2013). arXiv:1306.2232

40. Yu A. Simonov, Phys. At. Nucl. 79, 295 (2016). arXiv:1503.06616

41. M.A. Andreichikov, B.O. Kerbikov, E.V. Luschevskaya, YuA Simonov, O.E. Solovjeva, JHEP 1705, 007 (2017). arXiv: 1610.06887

42. A.M. Badalian, B.L.G. Bakker, YuA Simonov, Phys. Rev. D 75, 116001 (2007).arxiv:hep-ph/0702157

43. A.M. Badalian, Yu.A. Simonov, B.L.G. Bakker, arxiv:hep-ph/0610193

44. YuA Simonov, Phys. Rev. D 88, 025028 (2013). arXiv:1303.4952

45. YuA Simonov, Phys. At. Nucl. 79, 265 (2016). arXiv:1507.07569

46. K.A. Olive et al., (Particle Data Group) Chin. Phys. C38, 9 (2014)
47. YuA Simonov, Nucl. Phys. B 307, 512 (1988)

48. YuA Simonov, Z. Phys. C 53, 419 (1992)

49. YuA Simonov, J.A. Tjon, Ann. Phys. (N.Y.) 300, 54 (2002)

50. A.M. Badalian, B.L.G. Bakker, YuA Simonov, Phys. Rev. D 75, 116001 (2007). arxiv:hep-ph/0702157

51. M.A. Andreichikov, B.O. Kerbikov, V.D. Orlovsky, YuA Simonov, Phys. Rev. D 87, 094029 (2013). arXiv: 1304.2533

52. M.A. Andreichikov, V.D. Orlovsky, YuA Simonov, Phys. Rev. Lett. 110, 162002 (2013). arXiv: 1211.6568

53. R. Gatto, M. Ruggieri, Phys. Rev. D 83, 034016 (2011). arXiv: 1012.1291

54. D. Gomez Dumm, M.F. Izzo Villafañe, S. Noguera et al., arXiv: 1805.04597

55. S.S. Avancini, W.R. Tavares, M.B. Pinto, Phys. Rev. D 93, 014010 (2016). arXiv:1511.06261

56. S.S. Avancini et al., Phys. Lett. B 767, 247 (2017)

57. M. Coppola, D. Gomez Dumm, N.N. Scoccola, arXiv:1805.01549

58. YuA Simonov, Phys. Rev. D 88, 093001 (2013). arXiv:1308.5553

59. Yu A. Simonov, Phys. Rev. D 88, 053004 (2013). arXiv: 1304.0365 